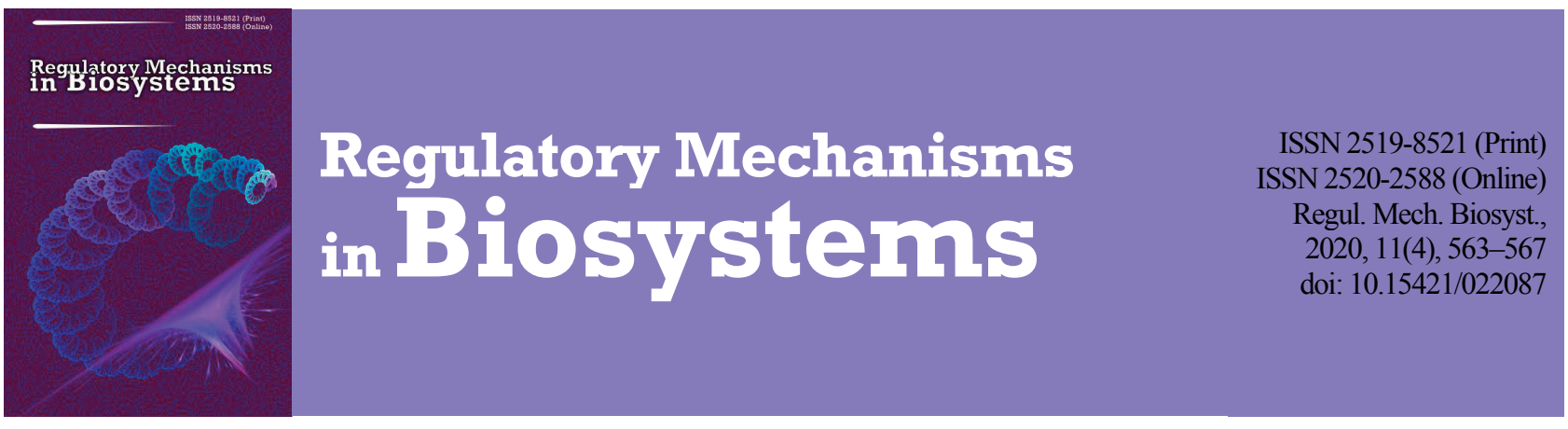

\title{
Use of polymerase chain reaction in verification and differential diagnosis of babesiosis pathogens
}

\author{
I. I. Torianyk*, O. M. Tymchenko*, M. O. Ostapets**, \\ N. A. Chygyrynska*, S. I. Pokhyl*, I. A. Kostyria*, I. V. Sorokina*** \\ *Institute Microbiology and Immunology named after I. I. Mechnikov of the National Academy of Medical Sciences of Ukraine, \\ Kharkiv, Ukraine \\ **National University of Pharmacy, Kharkiv, Ukraine \\ ***Kharkiv National Medical University, Kharkiv, Ukraine
}

Article info

Received 21.10.2020

Received in revised form 15.11 .2020

Accepted 17.11.2020

I. Mechnikov Institute of Microbiology and Immunology National Academy of Medical Sciences of Ukraine,

Pushkinska st., 14-16,

Kharkiv, 61057, Ukraine.

Tel.: +38-098-026-45-04.

E-mail:kamysh_in@ukr.net

National University of

Pharmacy, Kulykivska st., 12

Kharkiv, 61002, Ukraine.

Tel.: +38-095-372-81-48.

E-mail:

marina.ostapets.22@gmail.com

Kharkiv National Medical

University, Nauky av., 4

Kharkiv, 61022, Ukraine.

Tel.: +38-099-66-19-750

E-mail: soririna@gmail.com

\section{Introduction}

Recent decades have been marked by a significant enrichment of the arsenal of methods for detecting unicellular, objectifying ontogenetic parameters, biochemical processes of life (Mojtahed et al., 2020). Parasitologists today know more than 100 species of the genus Babesia (causative agents of babesiosis in mammals and birds) (Gray \& Weiss, 2008; Krause $\&$ Peter, 2019). The discovery of new species led to the renewal of classification of dominants, changes in taxonomy and significant loss of competence by the previously used classification of Babesia spp. (based on morphological differences of parasites, vectors of infectious transmission, ways of existence of the intermediate host) (Kim et al., 2007; Laha et al., 2015). The latter was objectively facilitated by the applied phenotypological and epidemiological criteria/parameters, their obvious instability, given the verification of the results and the subjectivity of the assessment. The modern view is focused on the molecular-biological (phylogenetic) version of the classification of Babesia species, their differentiation in accordance with the evolutionarily related relationship (Kjemtrup \& Conrad,
Torianyk, I. I., Tymchenko, O. M., Ostapets, M. O., Chygyrynska, N. A., Pokhyl, S. I., Kostyria, I. A., \& Sorokina, I. V. (2020). Use of polymerase chain reaction in verification and differential diagnosis of babesiosis pathogens. Regulatory Mechanisms in

Today, Babesia is recognized as one of the most common blood parasites in the world, which in terms of the number of cases ganis is second only to trypanosomes (the causative agent of African trypanosomiasis and Chagas' disease). These microorch can vary from asymptomatic, subclinical, mild or moderate influenza-like forms - to severe progressive disease (fulminant form) with fatal outcome. Thus, the latter determines the significant burden of babesia for the leading branches of medicine, veteriosis causative agents by the polymerase chain reaction (PCR) method. Blood, erythrocyte suspension, homogenized tick-carriers of diagnostics method was used in two formats - standard and multiplex (multi-primer). Multiple PCR testing of multiplex form using primers in model samples containing cells of different species of Babesia (B. microti, B. divergens, B. bovis, B. canis), allowed us to establish the level of reproducibility of the results of such studies, which ranged $94.6-96.4 \%$, to determine the level of venatorum). It is established that the advantages of the PCR-diagnostic method of babesiosis pathogens in the samples of the studied species identification, differentiation with apicomplex spores (Plasmodium falciparum, Toxoplasma). In view of the above, the PCR method is recommended for use in cases of persistent suspicion of babesiosis infection (in cases of negative results of microscopic/cytological studies, to identify asymptomatic, subclinical and chronic forms of babesiosis, verification of active invasion in seroposi-

Keywords: Babesia; verification; blood; homogenates of ticks; cultures of Babesia; polymerase chain reaction.

2006; Gray et al., 2010). According to the level of sequence similarity of the nucleotides of the small subunit ribosomal DNA gene (nuclear small subunit ribosomal DNA - NSS rDNA, synonym - 18S rDNA) Babesia are divided into 5 distinct groups (clades). This takes into account the (molecular-biological) specificity of strains with different geographical/ biotope origin of the primary DNA structure (both nucleotide sequences of $18 \mathrm{~S}$ rDNA and a number of other genes: ITSs) (internal transcribed spacers 1, 2), $\beta$-tubulin (beta tubulin), hsp70 (heat shock protein 70), SA1 (surface antigen 1), CCT7 (chaperonin-containing t-complex protein 1), PO (phosphoprotein O) (Beck et al., 2011; Solano-Gallego et al., 2016).

The above provisions contain obvious practical meaning, focused on a detailed analysis of epidemically/epizootically dominant genotypes of babesiosis pathogens, as an objective resource of a thorough evidence base for differential diagnosis of babesiosis, its effective therapeutic technologies (Vannier \& Krause, 2012; Ekpanyapong \& Reddy, 2020). The use of the PCR arsenal in the differential diagnosis of varieties of babesiosis pathogens (as a little-studied transmissible, hemoparasitic anthropozoonosis) is timely/relevant. 
Polymerase chain reaction (PCR) occupies a dominant position among molecular-genetic methods for diagnosing babesiosis infection (Gray \& Weiss, 2008; Zhou et al., 2014). PCR is characterized by a satisfactory speed of reproduction for obtaining research results with a higher level of sensitivity and specificity versus the microscopic and cultural methods (MM and CM, respectively) of detection of Babesia spp. (Herwaldt et al., 2003; Zhou et al., 2014). The level of sensitivity of the standard PCR format, determined during testing of model (Babesia-contaminated) blood samples makes it possible to reveal approximately $0.0001 \%$ of parasitaemia, and about 50-100 copies of genome fragments of these haemoparasites in $5.0 \mu \mathrm{M}$ of the tested blood sample when PCR is reproduced in the real-time format (Homel et al., 2000; Kim \& Epstein, 2001). Therefore, it is recommended to use PCR in practical medicine for diagnosing babesiosis, when its course is suspected in a patient, but microscopy of his/her blood smears gave a negative or controversial result. Besides, it is also recommended to use PCR in controlling biosafety of donor blood, revealing chronic and subclinical Babesia invasions, controlling effectiveness of antiparasitic therapy and perfecting identification of Babesia species, including discovery of their new representatives, etc. (Zhou et al., 2013; Fang \& McCullough, 2016). The principle, on which the use of PCR for diagnosing Babesia infection is based, consists in detection (owing to multiple amplification) of specific fragments of the genome (nucleic acids: DNA, RNA) of the causative agent in the examined clinical material samples (most commonly it is blood) from patients, thereby making it possible to draw a conclusion about the presence of the pathogens themselves in these specimens. Both species-specific and more universal genus-specific systems of primers are used for detecting/identifying Babesia spp. In the first case, DNA fragments of certain genes are detected, these fragments being specific and to some extent unique by the sequence of nucleotides for the given species of Babesia. In the second case, an amplification of DNA regions that are more universal (conservative) for Babesia spp. (most commonly $18 \mathrm{~S} \mathrm{rDNA}$ and SSU rDNA genes) is made with subsequent (if necessary) sequencing of the formed amplicons and identification (if there is any difference in their nucleotide sequences) of the species of causative agent (Beck et al., 2011; Solano-Gallego et al., 2016).

\section{Material and methods}

The study by the method of PCR was conducted with use of two formats (types) of reproduction of this reaction: the standard format (PRC $\mathrm{SF}$ ) and the multiplex (multiprimer) format (PRC MF). PRC SF was reproduced using commercial test systems (manufactured by "IsoGene Lab. Ltd", the Russian Federation) for detection of Babesia spp. and perfect identification of the parasite species (microscopic (MM), cultural $(\mathrm{CM})$ and biological methods (BM)) during verification of the protozoans in the examined samples (blood, erythrocyte suspensions, homogenates of ticks, cultures of Babesia) used for making experimental samples of complete cell-combined antigens as well as model samples, which were used for determining the specificity, sensitivity and reproducibility of PRC MF. Detection of the genus-specific fragments of the genome of Babesia ( $\mathrm{Ba}$ besia spp.) was performed with use of the diagnostic kit "The kit of reagents for amplifying DNA of Babesia spp., Gene Pak ${ }^{\mathbb{R}}$ PCR test", Cat. No. E 2130. For the purpose of species identification we used diagnostic kits "The kit of reagents for amplifying DNA of Babesia microti, Gene $\mathrm{Pak}^{\mathbb{B}} \mathrm{PCR}$ test", Cat. No. E 2129, "The kit of reagents for amplifying DNA of Babesia divergens, Gene Pak ${ }^{\mathbb{B}}$ PCR test", Cat. No. E 2126, and "The kit of reagents for amplifying DNA of Babesia canis, Gene Pak ${ }^{\mathbb{B}}$ PCR test', Cat. No. E 2127, respectively. The protocol of conducting of PCR SF has been standardised (IsoGene Lab. Ltd, 2010, the Russian Federation).

The flow chart of the development of the above variant of PCR envisages the following successively performed tasks: formation of a sample of certain fragments of the genome (target genes) of B. microti, B. divergens and $B$. venatorum (GenBank, USA: www.nebi.nlm.gov/Web/Gen Bank/index.html), European Molecular Biology Laboratory Data Library (EMBL, Europe: www.ebi.ac.uk/embl/index.html) and DNA Data Bank of Japan (DDBJ: www.nig.ac.jp/home.html); analysis of nucleotide sequences of the chosen genes with determination of their conservative and variable regions for modelling primer systems (PS); constructing of PS with determination of the length, primary structure and level of homology of oligonucleotides, their thermodynamic characteristics, presence of palindromic sequences, probability of the formation of hairpins and duplexes, position of Babesia on the DNA gene. The main task consisted in development of such PS (with at least three oligonucleotides, each of them being 18-24 nucleotides in length) for the multiplex PCR, whose use would make it possible, with a sufficient level of specificity, sensitivity and reproducibility of results of researches, to detect/identify simultaneously such Babesia species as B. microti and B. divergens $+B$. venatorum, which dominate in human pathology in Ukraine, with a discriminant difference in the size of the formed specific amplicons $>100$ base pairs (bp). In order to construct the authors' PS, a subbase of sequenced sequences of 18S rRNA gene of Babesia species B. microti, B. divergens, B. venatorum (all in all, $10^{7}$ sequences) and malaria plasmoids and toxoplasms (all in all, 24 sequences) was created from bioinformatic databases of nucleic acids GenBank, EMBL and DDBJ.

In order to analyze base pairs of $18 \mathrm{~S}$ rRNA gene in isolates of $B$. microti, $B$. divergens and $B$. venatorum, construct primers and assess their parameters, which are technologically important for reproducing multiplex PCR, we used application software package "Vector NTI Advance 11.0" (http://vector-nti.software.informer.com/11.0). Multiple alignment and its statistical analysis for defining conservative and variable regions of $18 \mathrm{~S}$ rRNA gene were made with help of software component module "AlignX". We used: module "Primer design" for constructing PS; "Oligo Analysis" for analyzing technologically important characteristics of primers; "Thermodynamic properties - Dimers and Hairpin Loops" for assessing thermodynamic properties of primers, the presence of palindromes, repetitions and possible formation of hairpins and dimmers; "Oligo Duplexes" for determining a probability of formation of duplexes. By results of software construction, a list of potentially suitable PS was made. Of them, a system with three oligonucleotides (BabUnF, BabMicR and BabDivR) was chosen; it had the highest automatically calculated rating -171 , thereby demonstrating the full conformity of characteristics of the above primers with the parameters set during their construction. Besides, the homology of a fragment of 18S rRNA gene in isolates of $B$. microti, B. divergens and B. venatorum to constructed primers Ba$\mathrm{bUnF}, \mathrm{BabMicR}$ and BabDivR as well as the specificity of these primers regarding the above region of the genome of Plasmodium spp. and Toxoplasma gondii were additionally checked using program module "Aligment" of program "MEGA4" (Mojtahed et al., 2020).

Further optimization of the above PS consisted in their "manual" correction. Here we took into account that primers would be annealed in regions of the single-stranded amplicon, which were homologous by their nucleotide composition, and PCR would take place with synthesis of new copies of the amplicon on condition that was choice of the optimum temperature for annealing primers (Tan), presence of homology of at least four 3'-end nucleotides in the primer-single-stranded amplicon complex and not more than two non-homologous (unpaired) nucleotides in the middle of the above complex. The possibility of some variability of nucleotide sequences in the middle region of primers makes it possible to construct them as "degenerate" ones, which makes it possible to detect fragments of the genome of different Babesia species during reproduction of multiplex PCR even in the presence of a certain variability in their nucleotide sequences. The negative result of multiplex PCR during an examination of a biomaterial sample was registered by the absence of streaks of an amplicon of any size or by the presence of an amplicon (or several amplicons) whose size differed from that of the proper control $\left(\mathrm{K}^{\dagger}\right)$ amplicon. A controversial result of multiplex PCR testing of a biomaterial sample and the necessity to repeat the study were stated in cases of obtaining visualization data of amplification products, which were insufficiently clear for analysis, including a poorly manifested fluorescent streak(s) of an amplicon(s) of the size, specific for B. microti and B. divergens $+B$. venatorum. Primers BabUnF, BabMicR and BabDivR were synthesized (in number of 205, 171 and 180, respectively; degree of purification "Salt Free") at the private enterprise "Laboratory of Leading Biotechnologies Neo-Gen" (Ukraine, www.neogene.com.ua). The negative result of multiplex PCR during an examination of a biomaterial sample was registered by the absence of streaks of an amplicon of any size or by the presence of an amplicon (or several amplicons) whose size differed 
from that of the proper control $\left(\mathrm{K}^{\dagger}\right)$ amplicon. A controversial result of multiplex PCR testing of a biomaterial sample and the necessity to repeat the study were stated in cases of obtaining visualization data of amplification products, which were insufficiently clear for analysis, including a poorly manifested fluorescent streak(s) of an amplicon(s) of the size, specific for $B$. microti and B. divergens $+B$. venatorum.

In carrying out multiplex PCR with primers BabUnF, BabMicR and BabDivR the authors used universal commercial kits manufactured by LLC "IsoGene Lab Ltd" (the Russian Federation, www.infobel.com/en/ .../isogen lab_ltd): "Diatom ${ }^{\mathrm{TM}}$ DNA Prep 100"; "GenePakT PCR Core"; "GenePak'TM DNA Ladder M50, M100"; a kit of reagents for detecting DNA by the method of electrophoresis in $15 \%$ agarose gel. The above kits of reagents were used in compliance with their manufacturer's instructions (Diatom ${ }^{\mathrm{TM}}$ DNA Prep 100, 2008; GenePak ${ }^{\mathrm{TM}}$ PCR Core, 2010). Determination of the main characteristics (levels of sensitivity, specificity and reproducibility of results) of the developed new variant of multiplex PCR for simultaneous detection/identification of $B$. microti, $B$. divergens and $B$. venatorum in the examined samples of biological material was performed by the way of testing (at least in three parallel repetitions) 82 model samples (intact blood and naturally infected or artificially contaminated blood samples). The model samples $(n=70)$ were of the following origin: 1) intact noninfected whole blood samples from human beings and animals $(\mathrm{n}=17)$ : human beings (Homo sapiens, $\mathrm{n}=5$ ), cattle (Bos taurus, $\mathrm{n}=3$ ), common vole (Microtus arvalis, $\mathrm{n}=3$ ), Syrian hamster (Mesocricetus auratus, $\mathrm{n}=3$ ), Mongolian gerbil (Meriones unguiculatus, $\mathrm{n}=3$ ); 2) blood samples a priori infected (the level of parasitaemia in the initial sample was defined with MM followed by several-fold calibration of model samples by their dilution from 10 to $10^{5}$ times) with Babesia $(\mathrm{n}=18)$, namely: Mesocricetus auratus, which contained B. microti with the level of parasitaemia from about 10 to about $0.0001 \%$ $(\mathrm{n}=6)$; Meriones unguiculatus, which contained $B$. divergens with the level of parasitaemia from about 5 to about $0.0005 \%(\mathrm{n}=6)$; Bos taurus, which contained B. bovis with the level of parasitaemia about 7.5 and $3 \%$ $(\mathrm{n}=3)$; Canis lupus, which contained B. canis with the level of parasitaemia about 25,14 and $6 \%(n=3)$.

In order to isolate DNA from model samples we applied the technology using the "Kit of reagents for universal sample preparation - "Diatom ${ }^{\mathrm{TM}}$ DNA Prep 100” (LLC 'IsoGene Lab.ltd.”, the Russian Federation) in compliance with the manufacturer's instruction. Samples of the isolated DNA were stored under refrigeration $\left(\mathrm{t}=-20^{\circ} \mathrm{C}\right)$.

The amplicons, formed as the result of multiplex PCR, were detected by the method of electrophoresis in 1.5\% agarose gel in Tris-borate buffer ("Kit of reagents for detecting DNA" manufactured by "IsoGene Lab.ltd.", the Russian Federation). $10 \mu \mathrm{L}$ of an amplified sample were put into a slot of a gel plate and electrophoresis was performed at a voltage level of $15 \mathrm{~V} / \mathrm{cm}$ during $20-30$ minutes. The gel was stained with $0.1 \%$ solution of ethidium bromide; the staining lasted 5 minutes. The results of multiplex PCR detection of specific fragments of 18S rRNA gene of $B$. microti, $B$. divergens and $B$. venatorum were analysed by visualization of the formed amplification products.

\section{Results}

At the present stage of this research the method of PCR detection of Babesia was used by us in order to verify (confirm) the results of revealing these parasites by MM and CM, namely for differentiating Babesia spp. from other intra- and extraerythrocytic formations, which are similar to these protozoans by their morphological characteristics. Therefore in the process of conducting researches by the method of PCR the latter was reproduced in the standard format using the commercial kit "The kit of reagents for amplifying DNA of Babesia spp., Gene Pak ${ }^{\circledR}$ PCR test" (manufactured by IsoGene Lab. Ltd., Moscow, the Russian Federation), which contains the genus-specific system of Bab primers and makes it possible to detect a DNA fragment (359 bp in size) of $18 \mathrm{~S} \mathrm{rDNA}$ gene of different species of protozoa of the Babesia genus.

All in all, the method of PRC was used for selective testing of 23 samples, which had the following origin: 3 samples of donor blood (potentially noninfected with Babesia) from a human being, a dog and a cow (the first blood sampling; respectively, samples $1 / \mathrm{HB} /-/ 1 /, 1 / \mathrm{D} /-/ 12 /$,
$1 / \mathrm{C} /-/ 6 /$ ), which gave $\mathrm{RBC}$ for culture feeding in vitro and served as control samples for conducting research by MM and CM; 5 samples from the dogs who suffered from tick bites and had clinical manifestations of a babesiosis-like disease (samples 1/D/-/1/, 1/D/-/5/, /1/D/-/7/, /1/D/-/9/, $1 / \mathrm{D} /-/ 11 /$ ) and 5 samples of erythrocyte suspensions (iRBC), which were received from the same samples and cultured in vitro; 5 blood samples from free-ranging cattle that suffered from multiple tick bites and had manifestations of haemoglobinuria (samples $1 / \mathrm{C} /-/ 1 /, 1 / \mathrm{C} /-/ 2 /, 1 / \mathrm{C} /-/ 3 /$, $1 / \mathrm{C} /-/ 4 /, 1 / \mathrm{C} /-/ 5 /)$ as well as 5 erythrocyte samples in vitro, which were received from the same samples and cultured in vitro.

The results of using the method of PCR for verification of detection of Babesia by MM and CM are shown in Table 1, an example of visualization of the results of PCR detection of Babesia spp. in the examined samples of different origin being presented in Figure 1. Analysis of the above findings demonstrated a general high level $\left(\mathrm{r}_{\mathrm{c}}=0.92\right)$ of the identity of results (both positive and negative) of revealing Babesia spp., received by MM, CM and the method of PCR. Here the level of correlation between the results of detection of haemoparasites by MM and PCR is characterized by $r_{c}=0.85$, the same index for the correlation between the results received by CM and PCR being $r_{c}=1.0$ (Table 1 ). Results of PCR detection of Babesia were characterized by a rather clear visualization; we did not observe any cases with doubtful values (Fig. 1): electrophoresogram tracks did not reveal any amplicons whose size would differ from that of the control amplicon $\left(\mathrm{K}^{+}\right)$, as well as a poorly expressed fluorescent amplicon band of the certain size ( $359 \mathrm{bp}$ ) that would complicate assessment of the above results and necessitate repetition of the relevant study.

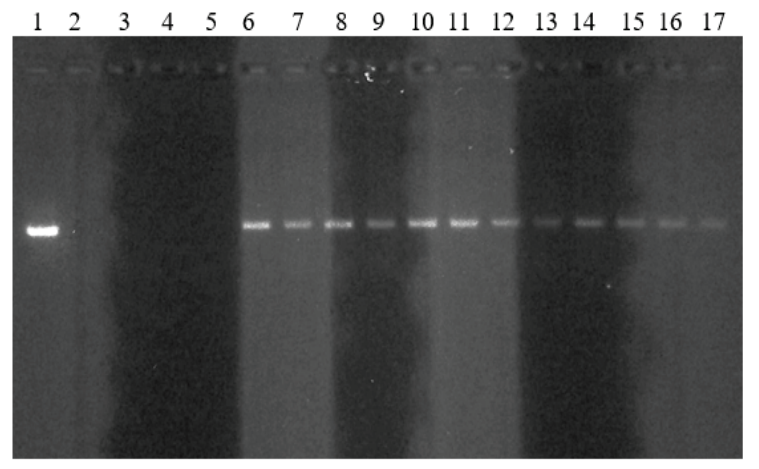

Fig. 1. An example of visualization of PRC detection of Babesia in samples of examined material using "The kit of reagents for amplifying DNA of Babesia spp., Gene Pak ${ }^{\circledR}$ PCR test" (Category No. E 2130) manufac-

tured by IsoGene Lab. Ltd. (Moscow, the Russian Federation):

1,2-a priori positive $\left(\mathrm{K}^{+}\right)$and negative $\left(\mathrm{K}^{-}\right)$control, respectively, of the commercial kit "The kit of reagents for amplifying DNA of Babesia spp., Gene Pak ${ }^{\circledR}$ PCR test" (Category No. E 2130) manufactured by “IsoGene Lab. Ltd." (Moscow, the Russian Federation); 3-5-a negative result of PCR of studying donor blood samples from, respectively, a human being 1/HB/-/1/, a dog 1/D/-/12/, a cow 1/C/-/6/, which served as control samples of blood, not infected with Babesia, and produced RBC; 6-17positive results of PCR, of them: $6-8$-three blood samples from dogs 1/C/-/1/, 1/C/-/5/, 1/C/-/9/, respectively; 9-12-four samples of iRBC after culturing the examined blood samples from dogs in vitro $1 / \mathrm{D} /-/ 1 /(5$ days in vitro), $1 / \mathrm{D} /-/ 5 /(4$ days in vitro), $1 / \mathrm{D} /-/ 7 /$ ( 7 days in vitro), $1 / \mathrm{D} /-/ 9 /$

(6 days in vitro), respectively; 13 - a blood sample from cattle 1/C/-/3/; 14-17 - four samples of iRBC after culturing the examined blood samples from cattle in vitro $-1 / \mathrm{C} /-/ 1 /, 1 / \mathrm{C} /-/ 3 /, 1 / \mathrm{C} /-/ 4 /, 1 / \mathrm{C} /-/ 5 /$, respectively

The third stage of our research envisaged studies for improvement of the method of PCR detection/indication of causative agents (Babesia spp.) of babesiosis in people with regard to new data concerning their most clinically significant varieties in the territory of Ukraine. Summarizing the above results of our research, the following generalizations were formulated. PCR helped to verify (confirm) results (selectively both negative and positive) of detecting Babesia spp. in samples of noninfected (donor) blood from people and animals and in blood samples with a high level of probable infection with haemoparasites.

The level of identity between the results of detecting Babesia by MM and the method of PRC is characterized by the value of the correlation 
ratio $\left(r_{c}\right)$ equal to 0.85 , this index between the results of revealing these haemoparasites by CM and PRC being $\mathrm{r}_{\mathrm{c}}=1.00$.

\section{Table 1}

Results of verification of revealing Babesia spp. by the method of PCR

\begin{tabular}{|c|c|c|c|}
\hline \multirow{2}{*}{$\begin{array}{c}\text { Origin of examined samples } \\
\text { (n-number of samples in the group) }\end{array}$} & \multicolumn{3}{|c|}{$\begin{array}{l}\text { Result of revealing Babesia } \\
\text { spp.: positive }(+) \text {, negative }(-)\end{array}$} \\
\hline & $\mathrm{MM}$ & $\mathrm{CM}$ & PCR \\
\hline \multicolumn{4}{|l|}{ Control blood samples from donors $(n=3)$ : } \\
\hline $\operatorname{man} 1 / \mathrm{HB} /-/ 1 /$ & - & - & - \\
\hline $\operatorname{dog} 1 / \mathrm{D} /-/ 12 /$ & - & - & - \\
\hline $\operatorname{cow} 1 / \mathrm{C} / 6 /$ & - & - & - \\
\hline \multicolumn{4}{|l|}{ Blood samples/iRBC from affected dogs $(n=5 / 5)$ : } \\
\hline $1 / \mathrm{D} / / / 1 /$ & + & + & $+/+$ \\
\hline $1 / \mathrm{D} /-/ 5 /$ & - & + & $+/+$ \\
\hline $1 / \mathrm{D} /-/ 7 /$ & + & + & $+/+$ \\
\hline $1 / \mathrm{D} /-/ 9 /$ & + & + & $+/+$ \\
\hline $1 / \mathrm{D} /-/ 11 /$ & + & + & $+/+$ \\
\hline \multicolumn{4}{|l|}{ Blood samples/iRBC from affected cattle $(n=5 / 5)$ : } \\
\hline $1 / \mathrm{C} / 1 /$ & + & + & $+/+$ \\
\hline $1 / \mathrm{C} / 2 /$ & - & - & $-1-$ \\
\hline $1 / \mathrm{C} / 3 /$ & + & + & $+/+$ \\
\hline $1 / \mathrm{C} / 4 /$ & + & + & $+/+$ \\
\hline $1 / \mathrm{C} / 5 /$ & + & + & $+/+$ \\
\hline
\end{tabular}

When "The kit of reagents for amplifying DNA of Babesia spp., Gene Pak ${ }^{\mathbb{R}}$ PCR test" (Category No. E 2130) manufactured by "IsoGene Lab. Ltd.", (Moscow, the Russian Federation) is used, the results of PRC detection of Babesia are characterized by a clear visualization of amplification products, thereby making it possible to determine its positive or negative result unambiguously.

\section{Discussion}

Nowadays among the methods of studying babesiosis the dominant position belongs to PCR, the use of its reserves in determining the etiopathogenesis of the disease - an undoubted laboratory criterion (Criado-Fornelio et al., 2003; Gray et al., 2010). PCR testing using primers BabUnF, BabMicR and BabDivR in model samples containing cells of different species of Babesia (B. microti, B. divergens, B. bovis, B. canis) opens up prospects for a refined level setting (94.6-96.4\%) reproducibility of research results, determination of PCR sensitivity in the detection/identification of human pathogenic Babesia (B. microti, B. divergens and B. venatorum). It makes sense to use this study plan as an evidence base for PCR multiplex format for detection/identification in biomaterial samples of several dominant species of Babesia (B. microti, B. divergens $+B$. venatorum pathogenic to humans with specificity levels of $100 \%$, sensitivity about $10^{4}-10^{5}$ pathogen cells in $\mathrm{ml}$ of the sample and reproducibility of test results $\geq 95 \%$ ). An additional positive point, which is repeatedly discussed in the development of PCR diagnostics, is the prospect of achieving a high level of discriminant difference in the size of the specific amplicons ( $>140 \mathrm{bp}$ ) under the conditions of detection/identification of B. microti (288 bp) and B. divergens + B. venatorum (141/142 bp).

The advantages of the method of PCR-diagnostics of babesiosis pathogens in the samples of the studied biomaterial experts are: the speed of research in real time (2-4 hours), high sensitivity, specificity, reproducibility of Babesia detection results $(\mathrm{P}<0.05)$, prospects of species identification, differentiation with apicomplex spores (Plasmodium falciparum, Toxoplasma). The convenience of PCR reproduction technology, the possibility of a unified approach in the use of consumables and tools are recognized (Parija et al., 2015; Solano-Gallego et al., 2016).

In view of the above, the PCR method is recommended by specialists for use in cases of persistent suspicion of babesiosis (in case of negative microscopic/cytological results, to identify asymptomatic, subclinical and chronic forms of babesiosis, verification of active invasion in seropositive individuals and Babesia species and their differentiation).

However, one should also consider the inherent shortcomings of this method. Among the latter are the high cost of equipment and reagents, strict research conditions to prevent "accidental" contamination with previously synthesized amplicons and obtaining of false-positive results. A major problem remains the impossibility of using PCR (except for real- time reverse transcription) to distinguish between viable and intact parasite cells, which makes it difficult to use this method for operational control of the effectiveness of antiparasitic therapy. A significant obstacle to wide practical application is the lack of standardized commercial test systems and validated research protocols available in European countries (including Ukraine) for the simultaneous detection/identification of the most relevant species causing babesiosis in humans: $B$. divergens, $B$. venatorum and B. microti (Herwaldt et al., 2002; Krause Peter, 2019).

\section{Conclusion}

According to the results of testing 70 samples of biological material, it was found that the PCR method using rhodospecific commercial JV Bab provided a high frequency $(\mathrm{P}<0.05)$ detection of Babesia. Pathogens in $27.7 \%$ of these samples of biological material were determined by PCR with a commercial rhodospecific system of Babesia primers. The latest exceeded $(\mathrm{P}<0.05)$ the total frequency $(10.0 \%)$ of detection/identification of Babesia species B. microti, B. divergens and B. canis PCR standard format using commercial species-specific primer systems Bmi, Bdi, Bca at the level of identification of positive and negative reaction results on the index $r_{c} 0.44,0.49,0.60$, respectively.

The studies can be used as an evidence base for PCR multiplex format for detection/identification in biomaterial samples of several dominant species of Babesia pathogenic to humans ( B. microti, B. divergens + B. venatorum) with levels of specificity of $100 \%$, sensitivity about $10^{4}-$ $10^{5}$ pathogen cells in one $\mathrm{mL}$ of the sample and reproducibility of test results $\geq 95 \%$. In addition, an additional positive characteristic of the developed PCR MF is a fairly high level of discriminant difference in the size of the specific amplicons ( $>40 \mathrm{bp}$ ) under the conditions of detection/identification of B. microti (288 bp) and B. divergens + B. venatorum (141/142 bp).

We are grateful to Doctor of Medical Sciences Oleksiy P. Bilozyerov (State Institution "Institute of Dermatology and Venerology of the National Academy of Medical Sciences of Ukraine", Kharkiv, Ukraine) for his help in registration of PCR results.

\section{References}

Beck, R., Vojta, L., Ćurković, S., \& Mrljak, V. (2011). Molecular survey of Babesia microti in wild rodents in Central Croatia. Vector Borne Zoonotic Diseases, 11, 81-83.

Criado-Fornelio, A., Martinez-Marcos, A. Buling-Saraña, A., \& Barba-Carretero, J. C. (2003). Presence of Mycoplasma haemofelis, Mycoplasma haemominutum and piroplasmids in cats from southern Europe: A molecular study. Veterinary Microbiology, 93, 307-317.

Ekpanyapong, S., \& Reddy, K. R. (2020). Fungal and parasitic infections of the liver. Gastroenterology Clinics of North America, 49(2), 379-410.

Fang, D. C., \& McCullough, J. (2016). Transfusion-transmitted Babesia microti. Transfusion Medicine Reviews, 30(3), 132-138.

Gray, J. S., \& Weiss, L. M. (2008). Babesia microti. In: Khan, N. (Ed.). Emerging protozoan pathogens. Taylor and Francis, Abingdon. Pp. 303-349.

Gray, J. S., Zintl, A., \& Hildebrandt, A. (2010). Zoonotic babesiosis: Overview of the disease and novel aspects of pathogen identity. Ticks and Tick-Borne Diseases, $1,3-10$.

Herwaldt, B. L., Cacciò, S., Gherlinzoni, F., Aspock, H., Slemenda, S. B., Piccaluga, P., Martinelli, G., Edelhofer, R., Hollenstein, U., Poletti G., Pampiglione, S., Loschenberger, K., Tura, S., \& Pieniazek, N. J. (2003). Molecular characterization of a non-Babesia divergens organism causing zoonotic babesiosis in Europe. Emerging Infectious Diseases, 9(8), 942-948.

Homer, M., Delfin, J. I. A., \& Telford, F. (2000). Babesiosis. Clinical Microbiology Reviewer, 13(3), 451-469.

Kim, A. C., \& Epstein, M. E. (2001). Coinfection with Babesia microti and Borrelia burgdorferi. Infection Medicine, 18, 271-276.

Kim, J. Y., Cho, S. H., \& Joo, H. N. (2007). First case of human babesiosis in Korea: Detection and characterization of a novel type of Babesia sp. (KO1) similar to ovine Babesia. Joumal of Clinical Microbiology, 45(6), $2084-2087$.

Kjemtrup, A. M., \& Conrad, P. A. (2006). A review of the small canine piroplasms from California: Babesia conradae in the literature. Veterinary Parasitology, $138,112-117$.

Krause Peter, J. (2019). Human babesiosis. International Joumal for Parasitology, 49(2), 165-174. 
Laha, R., Das, M., \& Sen, A. (2015). Morphology, epidemiology, and phylogeny of Babesia: An overview. Symposium, 5(2), 94-100.

Mojtahed, A., Bates, D. D. B., \& Hahn, P. F. (2020). Splenic findings in patients with acute babesiosis. Abdominal Radiology, 45, 710-715.

Parija, S. C., Dinoop, K. P., \& Venugopal, H. (2015). Diagnosis and management of human babesiosis. Tropical Parasitology, 5(2), 88-93.

Solano-Gallego, L., Sainz, Á., \& Roura, X. (2016). A review of canine babesiosis: The European perspective. Parasites Vectors, 9, 336.
Vannier, E., \& Krause, P. J. (2012). Human babesiosis. The New England Journal of Medicine, 366(25), 2397-2407.

Zhou, X., Li, S. G., \& Chen, S. B. (2013). Co-infections with Babesia microti and Plasmodium parasites along the China-Myanmar border. Infectious Diseases Poverty, 2(1), 24.

Zhou, X., Xia, S., Huang, J. L., Tambo, E., Zhuge, H. X., \& Zhou, X. N. (2014). Human babesiosis, an emerging tick-borne disease in the People's Republic of China. Parasites and Vectors, 7, 509. 